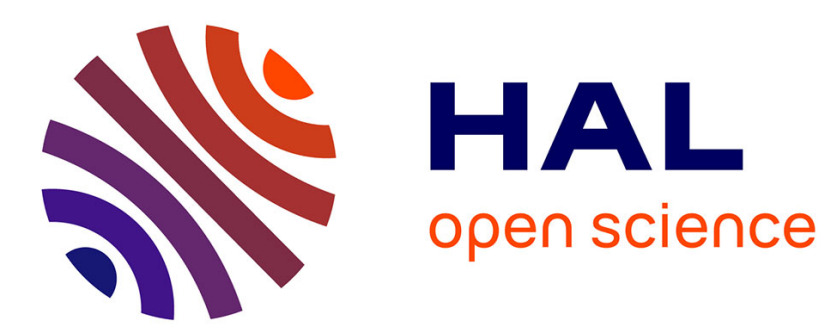

\title{
Serendipitous Learning Fostered by Brain State Assessment and Collective Wisdom
}

\author{
Stefano A. Cerri, Philippe Lemoisson
}

\section{To cite this version:}

Stefano A. Cerri, Philippe Lemoisson. Serendipitous Learning Fostered by Brain State Assessment and Collective Wisdom. BFAL 2020 - 2nd International Conference on Brain Function Assessment in Learning, Oct 2020, Heraklion, Crete, Greece. pp.125-136, 10.1007/978-3-030-60735-7_14 . lirmm02960627

\section{HAL Id: lirmm-02960627 https://hal-lirmm.ccsd.cnrs.fr/lirmm-02960627}

Submitted on 7 Oct 2020

HAL is a multi-disciplinary open access archive for the deposit and dissemination of scientific research documents, whether they are published or not. The documents may come from teaching and research institutions in France or abroad, or from public or private research centers.
L'archive ouverte pluridisciplinaire HAL, est destinée au dépôt et à la diffusion de documents scientifiques de niveau recherche, publiés ou non, émanant des établissements d'enseignement et de recherche français ou étrangers, des laboratoires publics ou privés. 


\title{
Serendipitous Learning Fostered by Brain State Assessment and Collective Wisdom
}

\author{
Stefano A. Cerri ${ }^{1,2,3(\bowtie)}$ and Philippe Lemoisson ${ }^{4,5}$ \\ 1 DKTS: Digital Knowledge Technologies Services SRL, Via Ampère 61/a, 20131 Milan, Italy \\ sacerri@didaelkts.it \\ 2 FBK: Fondazione Bruno Kessler, Trento, Italy \\ 3 LIRMM, Univ Montpellier and CNRS, 161 Rue Ada, 34095 Montpellier, France \\ 4 CIRAD, UMR TETIS, 34398 Montpellier, France \\ philippe. lemoisson@cirad. fr \\ 5 TETIS, Univ Montpellier, AgroParisTech, CIRAD, CNRS, IRSTEA, Montpellier, France
}

\begin{abstract}
Serendipitous discovery, invention or artistic creation are among the most exciting and utmost relevant phenomena strongly related to human learning. At the moment, there are very few measurable criteria helping to understand and foster serendipity. In other papers [1-3] we have presented, discussed and exemplified a new paradigm/model/method/system/environment - called ViewpointS that represents our efforts to overcome many current existing limitations in generic Information Systems or search engines (e.g.: Google) as well as in other social media (e.g.: recommender systems) offering information retrieval solutions based on the proximity of available resources. We also have also exposed how ViewpointS may facilitate serendipitous discovery in an unprecedented way. In this paper, we wish to further motivate this last conjecture by proposing to explore two main research directions that did not convey sufficient attention by previous researchers (in particular those active in recommender systems): 1. assessing brain states in order to understand and forecast serendipitous human learning events triggered by emotions; 2. enhancing collective wisdom, since Human-Computer Interactions do not occur today between a human and a single machine (or algorithm), but within a community of humans and machines that continuously update "knowledge" beyond the scene. Both directions (assessment of brain states, collective wisdom) are currently on separate ways; we propose to combine them within one unified approach called ViewpointS.
\end{abstract}

Keywords: Collective intelligence $\cdot$ Human learning $\cdot$ Serendipity triggered by brain states $\cdot$ Socially driven serendipity 


\section{Introduction}

This paper aims to identify, motivate and document some necessary requirements for the success of a research challenge ${ }^{1}$ that is ongoing since many years: understanding and forecasting serendipitous learning.

Understanding human learning is one of the priorities of the next century if we consider the complexity of the subject and the impact that any progress may have.

In its essence, human learning may occur as a result of interactions with the external world ${ }^{2}$, either because an external agent "communicates" some information, concept, relation, attribute, way-to-do (learning by being told) or because the subject constructs some chunk of knowledge (concept, how-to-do, relation, property, ...) in his/her mind. This latter event may be associated to the known theories of human learning by Piaget or Vygotsky. Both events occur by interaction. It has been demonstrated that animals (and humans) that are not exposed to a rich interactive environment do not learn as well as those that are immersed in knowledge-rich interactive scenarios.

Independently from "how" learning occurs, we may ask a question about "what is" human learning. Under the hypothesis that at higher cognitive levels the natural location representing and producing human cognitive behavior - including learning - is the brain, we are aware that the brain is not a computer, and concepts, relations, procedures, facts, ... are all represented and stored in a highly distributed and temporally mutable way [4]. Therefore, we may for the moment only reason by looking at individual's cognitive behavior before and after a learning event. We come to the conclusion that learning is a "state change" measurable only - for the moment - by looking at its effects: changes in human knowledge, know-how, reaction times to questions, ... As it was stated by various authors in the Web Science movement, the Web is the most interesting laboratory ever invented for studying Human-Computer and Human-Human interactive phenomena.

Considering self-awareness, learning may occur when humans are aware: they wish to learn, they positively engage their resources in order to reach the goal to learn: it is the case of the school, learning to play an instrument, to improve the performance in a physical activity, such as a sport, etc. However, most of human learning occurs when the learner is not aware i.e.: informally or incidentally. Serendipity, explored in the following section, represents not only a phenomenon of informal learning useful for the subject, but one of extreme importance as it impacts the whole community.

In Sect. 2 we give a short, historically supported introduction to the concept of serendipity. In Sect. 3 we discuss some early research in linking Information retrieval and serendipity, including general remarks and recent analogies about the interest of serendipitous behavior even in service-oriented computing. Section 4 is dedicated to some recent serendipity research in recommender systems. The three "introductory"

\footnotetext{
1 This paper has the objective to stimulate reflections about an ambitious, but equally modest and feasible, collaborative, large, long term research project for understanding, forecasting and stimulating serendipitous learning, i.e.: discovering, inventing, creating. At the same time we claim this project to be a concrete step towards digital sovereignty since it enables to personalize -thus control and trust- effectively the individual and collective information spaces, differently from current search engines, social networks and recommender systems.

${ }^{2}$ From: https://en.wikipedia.org/wiki/Learning: Humans learn before birth and continue until death as a consequence of ongoing interactions between people and their environment.
} 
Sects. 2, 3 and 4 are rich of excerpts from the literature in order to justify the primary interest of serendipity studies today and the minimal requirements for stimulating human serendipitous learning. These are identified to consist of measurements of emotions brain state assessment in the title- and interactions within a knowledge space fed by trusted peers and regulated by a subjective topology (proximity of resources: concepts, agents and documents) - collective wisdom in the title-. Within this frame, the ViewpointS paradigm is briefly introduced in Sect. 5 ending with an annotated, toy example of a process facilitating serendipitous discoveries, inventions or creations. Section 6 concludes the paper.

\section{The Concept of Serendipity}

One of the early authoritative sources [5] reports that: Serendipity can be defined as the supposed capacity to discover, invent, create or imagine something important without deliberately being in quest for it. If somebody discovers, invents, creates or imagines something of general value, without being deliberately in quest of it then his finding can be called serendipitous. So, we speak about a serendipitous discovery, invention, creation or thought. Later, in another paper [6] where the author identifies 17 serendipity patterns, he specifies: If I define true serendipity as the art of making an 'unsought finding', what do I mean by a 'finding'? I speak of a 'finding' when two or more elements (observations, hypotheses, ideas, facts, relations or insights) are combined originally, for the finder or anybody, to something new and true (science), new and useful (technology), or new and fascinating (arts). The number, quality and interest of serendipity examples described 30 years ago is astonishing.

A "recent" paper [7: archived from 2014 and updated till 2019] reporting about several projects (British but also EU FET) has a rather complete bibliography about serendipity research and systems (more than 100 papers quoted). While in their projects the goal was to define a formal model of serendipity and an associated creative computational system, we focus here on understanding, forecasting and facilitating human serendipitous learning behavior: we believe to have different goals and preconditions "what" and "why" - and different means - "how" -, even if their work is highly valuable for us as well.

Another convincing statement - about progress as reported in scientific papers from Pek Van Andel [6], is the following one: In general the role of serendipity in science, technology and art is underestimated. This is mainly and unintentionally caused by the way we rationalize "a posteriori" about theoretical and experimental research and its results, when we publish. The not strictly rational, chronological or searched components (like chance, fortuitous, accidental, surprising, unsought (n)ever dreamt of, unknown, etc.), which have led to these results are therefore underestimated and sometimes even banned from the theater and totally hidden behind the décor. The next step is that pure rationality becomes the norm, not only regarding the results, but also regarding everything that has led to these results. Scientists then report their results as 
following directly and logically ${ }^{3}$ from their initial hypothesis, omitting possibly crucial ( $p$ seudo)serendipitous events. Reading and interpreting such articles as 'the inside story' about the discovery, can unwillingly brainwash the researcher in such a way and to such an extent that he neglects during his own research the flowers along the road that can form a nicer bouquet than those he is looking for. This can cause a loss of serendipity: the aim and/or plan spoils the journey. A successful researcher or manager has one open eye for sought findings and another open eye for unsought findings.

So, in the following we feel encouraged to talk about serendipity in information systems, in particular recommender systems, even if we did not explore the whole gigantic literature about those systems, but just a minimal part of it, necessary and sufficient for our goals. Our rationality is limited, we admit, but accompanied by very well founded feelings and curiosity about our conjectures.

These feelings started years ago when the Web was in its early stage [8]. The research on ViewpointS [1-3] was motivated by an attempt to bypass the "intractable" problem of "domain, time and author's dependent ontologies" and respect the conviction that humans in the loop are a much better source of intelligence. Its main result was a unified (logical, statistical, social) and personalized context of knowledge construction. The serendipity potential was a fortuitous side effect of very different initial goals.

\section{Serendipity in Information Retrieval}

Another early author [9] gives clues to understand the interest (and the resistances) to study, accept and facilitate serendipity in information systems: The acquisition of information is generally thought to be deliberately sought using a search or query mechanism or by browsing or scanning an information space. People, however, find information without seeking it through accidental, incidental or serendipitous discoveries, often in combination with other information acquisition episodes. The value of this phenomenon to an individual or an organization can be equated with the impact of serendipitous breakthroughs in science and medicine. Although largely ignored in information systems development and research, serendipitous retrieval complements querying and browsing, and together they provide a holistic, ecological approach to information acquisition and define the key approaches to a digital library. Further: In essence, there are three ways in which people acquire information, each of which should be supported by a digital library: 1. from the search for information about a well-defined and known object(s); 2. from the search for information about an object that cannot be fully described, but will be recognized on sight; and, 3. from the accidental, incidental, or serendipitous discovery of an object. Systems that support the first prompt users for search terms and keywords and provide options for manipulating the query and the result. Hypermedia, visualization and menu-driven systems that provide views and overviews of the data facilitate the second. In both cases, the next step taken, e.g., next index term selected, next page

\footnotetext{
${ }^{3}$ Rationalism and empiricism exist and balance each other since centuries in the philosophy of Science, as much as idealism and materialism. In the brain, logical thinking is associated to emotions; in Society it occurs probably the same. It is not rational to negate the interest of serendipitous events and the associated processes.
} 
examined or information node accessed has a conceptual and/or a semantic relationship with the previous one. In the first, that relationship is predetermined by the system's response to the query; while in the second, the relationship is enabled for the most part by directed pathways through the system. While the process of information seeking is more complex than this, the outcome is generally the same: to locate the information object; this is a highly purposive task. The third type, the serendipitous approach, is a type of information seeking that is not traditionally examined in information retrieval research and has received little attention by both developers and researchers. The same author, under the heading "facilitating serendipity" explains: In the case of my study, the serendipitous activity was stimulated and triggered by a list of articles that bore some degree of similarity to the one currently displayed. Further, she quotes another aspect of serendipity: creativity: Just as the creative process must result in new ideas that are intelligible to the creator and understood in terms of prior knowledge (Boden, 1996 ${ }^{4}$ ), so too must the results of a chance encounter. Apparently, there is a need to link the new "idea" or "concept" to previous knowledge, a conjecture very similar to Vygotsky's "law of proximal development". E.G. Toms concludes: Serendipitous retrieval demands approaching information retrieval in an unorthodox manner, one that does not tightly couple the explicit match of query with result, but instead takes a fuzzy approach to the problem. Yet, there is no link with "the brain's state" neither with any "social context".

Other authors [10] have attempted to propose an empirically founded model for serendipity that emerged from several interviews: Our empirically-grounded process model of serendipity (Fig. 1 below, by courtesy of the authors) focuses on the mental connection that is sparked by circumstances that are to some extent unexpected. A new connection is made between an informational or non-informational need and a 'thing' (e.g. person, event, place, information, object) with the potential to address the need. The circumstances that led to the connection are subsequently (and subjectively) considered to have involved an amount of unexpectedness. The making of the connection itself is subjectively considered to have involved an amount of insight.

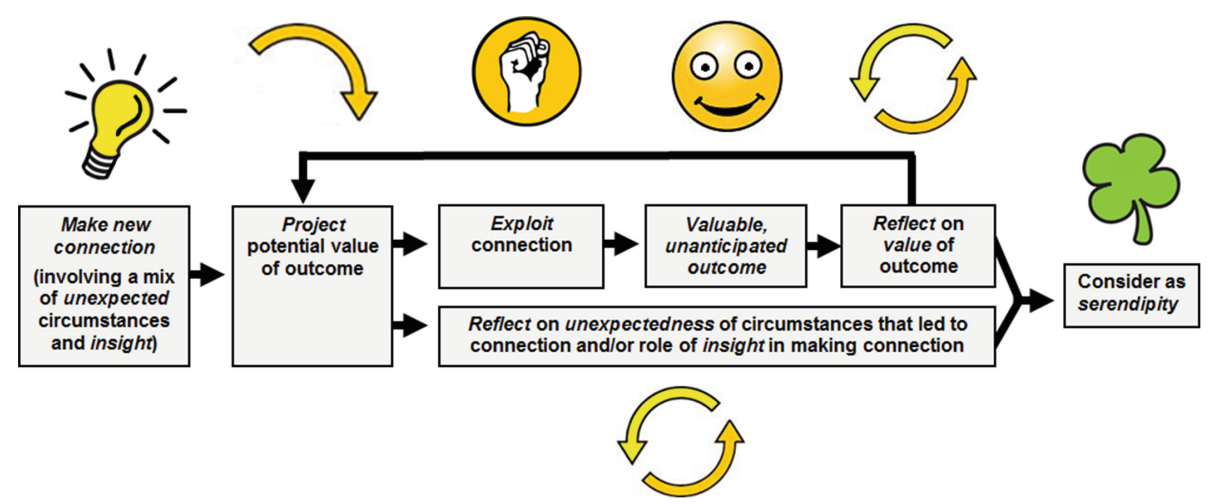

Fig. 1. $<$ An $>$ empirically-grounded process model of serendipity $<$ from: [10] $>$

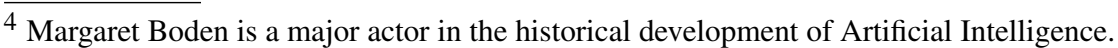


The model is the result of a long empirical research based on interviews that not only produced the model but also a classification of different serendipity events.

However, differently from Toms, the authors do not offer suggestions in this paper about how to organize information spaces in order to support serendipity.

In a subsequent paper [11] there is finally some clear roadmap about how to proceed: While initial discussions on accidental information discovery in digital environments focused on concerns about the loss of serendipity as a result of digitalization, most current discussions accept that digital information environments can and, where useful, should create opportunities for serendipity. As Lori McCay-Peet notes in her essay "Digital Information Environments That Facilitate Serendipity", "It is no longer about whether technologies are helping or hurting serendipity. That question is too broad and not helpful." A more helpful question, and one that is answered in this chapter, is "how can we design digital information environments to (best) cultivate serendipity?" This is also our concern.

The message has been adopted by other researchers [12] that conclude their work with a strong support to "prioritization": In this paper we have extended our earlier model for information seeking in large-scale digital libraries with new strategies and modes of contextual browsing, showing their consistency with behaviors supporting serendipitous discovery set out by Makri et al. We have described how contextual browsing requires prioritization to remain feasible in large-scale scenarios and have proposed the application of contextual similarity metrics to this purpose. However, the system "proof of concept" they used (Compage) did neither capitalize from the dynamic behavior of communities, nor from the assessment of brain mental states.

The interest of serendipity is also addressed recently by other authors [13] that relate serendipity to service-oriented computing: In service-oriented computing, software applications evolve dynamically to meet their goals through discrete reconfigurations triggered by the need for an external resource and realized through a three-fold process of discovery, selection and binding of service modules. To model creative systems, we regard concepts as modules and concept discovery as service discovery.

The analogy with human serendipity is striking: One salient feature of this approach is the unpredictability of the interactions between service entities. The continuous change in the availability of modules places us in a dynamic context where chance plays an important role in the evolution of systems. The selection of a most promising concept (provider of a resource) is based on the semantic compatibility of specifications. Because applications change over time, the evaluation of concepts can change as well, generating a focus shift.

\section{Serendipity, Recommender Systems and Emotions}

A rather recent study [14] addresses the issue not just of information retrieval, rather of recommender systems, a kind of proactive systems. The essence is contained in their abstract: Most recommender systems suggest items similar to a user profile, which results in boring recommendations limited by user preferences indicated in the system. To overcome this problem, recommender systems should suggest serendipitous items, which is a challenging task, as it is unclear what makes items serendipitous to a user and how 
to measure serendipity. The concept is difficult to investigate, as serendipity includes an emotional dimension and serendipitous encounters are very rare. In this paper, we discuss mentioned challenges, review definitions of serendipity and serendipity-oriented evaluation metrics. The goal of the paper is to guide and inspire future efforts on serendipity in recommender systems.

The authors finally admit that: Relevance of an item for a user might depend on user mood <...omissis...> This contextual information is difficult to capture without explicitly asking the user. As serendipity is a complex concept, which includes relevance $<\ldots>$, this concept depends on the current user mood in a higher degree. An emotional dimension makes serendipity unstable and therefore difficult to investigate $<\ldots>$.

The first conclusion of this study is that the system should recommend serendipitous items, a challenging task; because items similar to a user profile result in boring recommendations. The second is that serendipity depends on the emotional state of the subject that is not only hard to evaluate by interviewing the subjects, but also unstable.

In [15] the authors propose "a theoretical model of surprise" (in recommender systems) that includes the following properties: surprise must be subjective, surprise must be dynamic, surprise is related to the notion of distance. The paper includes several measures of surprise and a list of specific and recent references. In the scenarios there is a clear exploitation of collective behavior (the Movie Lens dataset used contains 3883 items, 6040 users and over 1 million ratings) but no suggestion about the influence of emotions in "surprise", thus in serendipity.

Taking the challenge indicated by these authors, we aim to suggest "serendipitous items" that should have three properties:

1. be linked to the previous user knowledge and experience;

2. be dependent from the user's emotional state;

3. be exciting and new for the user, not boring.

\section{ViewpointS: Emotions and Collective Wisdom for Serendipity}

This section first briefly recalls the Viewpoint $S^{5}$ framework and formalism for building collective knowledge in the metaphor of the brain (a detailed description can be found in $[1-3]$ but also $[17,18]$ and then illustrates through an imaginary toy example the specific interest of ViewpointS for facilitating serendipitous learning.

In the ViewpointS approach, the "neural maps interconnected by beams of neurons" [4] are transposed into a graph of "knowledge resources (Agents, Documents, Topics) interconnected by beams of viewpoints". Agents (Human or Artificial) are the only active resources. The "systems of values" of the Agents influence the viewpoints they emit, but also the way they interpret the graph.

We call knowledge resources all the resources contributing to knowledge: Agents, Documents and Topics. We call viewpoints the links between knowledge resources.

$\overline{5}$ This section is a revised, annotated version of the similar section of another paper. On purpose, we use the same very simple example in order to support quite different conjectures about the properties of ViewpointS; in this case serendipity. 
Each viewpoint is a subjective connection established by an Agent (Human or Artificial) between two knowledge resources; the viewpoint $\left(\mathrm{a}_{1},\left\{\mathrm{r}_{2}, \mathrm{r}_{3}\right\}, \theta, \tau\right)$ stands for: the Agent $a_{1}$ believes at time $\tau$ that $r_{2}$ and $r_{3}$ are related according to the emotion carried by $\theta$. Agents entitled to feed viewpoints are selected by the user according to his/her preferences (they are trusted Agents).

We call Knowledge Graph (KG) the bipartite graph consisting of knowledge resources and viewpoints. Given two knowledge resources, the aggregation of the beam of all connections (viewpoints) linking them can be quantified and interpreted as a proximity (or, inversely, as a distance). We call perspective the set of rules (defined by the user) implementing this quantification by evaluating each viewpoint and then aggregating all these evaluations into a single value: the single value is associated to the two resources. The graph resulting from the transformation activated on the KG by the rules of the perspective is called Knowledge Map; since the KG is continuously updated by trusted Agents, the KM is also continuously updated.

Notice that there are two sources of subjectivity: the choice of the trusted Agents that feed the KG (preference) and the rules transforming the $\mathrm{KG}$ into the $\mathrm{KM}$ (perspective). This double subjectivity represents the user's system of values. Both are chosen by the "Agent-consumer" of the information, not by a third-part "Agent-producer" such as a Google or Amazon algorithm. Each time an Agent wishes to exploit the knowledge acquired from the community of trusted Agents, he does so through his own subjective perspective which acts as an interpreter.

Tuning a perspective may for instance consist in giving priority to selected trustworthy agents, or to the most recent viewpoints, or to the viewpoints issued from the logical paradigm. This clear separation between the storing of the traces (the viewpoints) and their subjective interpretation (through a perspective) protects the human agents involved in sharing knowledge against the intrusion of third-part algorithms reifying external systems of values, as it is the case for most recommender systems. Adopting a perspective yields a tailored KM: knowledge map where distances can be computed between knowledge resources, i.e. where the semantics emerge from the topology as well as from our own subjective system of values expressed by the perspective and by the choice of the trusted Agents (preference).

To illustrate this, we develop below an imaginary case where learners have to select resources inside an Intelligent Tutoring System (ITS) to which a Knowledge Map is associated. They wish to learn about the topic 'apple' and from step1 to step4 the learners adopt a 'neutral' perspective which puts in balance all types of viewpoints (issued from the logical or mining paradigms, or from the emotions of the learners). However, at step5, B chooses a perspective discarding his own viewpoints in order to discover new sources of knowledge. What is figured in the schemas (see Fig. 2) is not the KG itself, but the KM resulting from the perspectives; in these maps, the more links between two resources, the closer they are.

Step1 illustrates the initial state of the knowledge. A, B and C are co-learners of an ITS $^{6}$ (linked as such within the logical paradigm: they belong to the same group); the blue arrows represent their respective systems of values, which play a key role both in the choice of perspectives and in the emission of viewpoints (Agents trusted to feed the

6 This is obviously an extremely simplified tutoring system! 

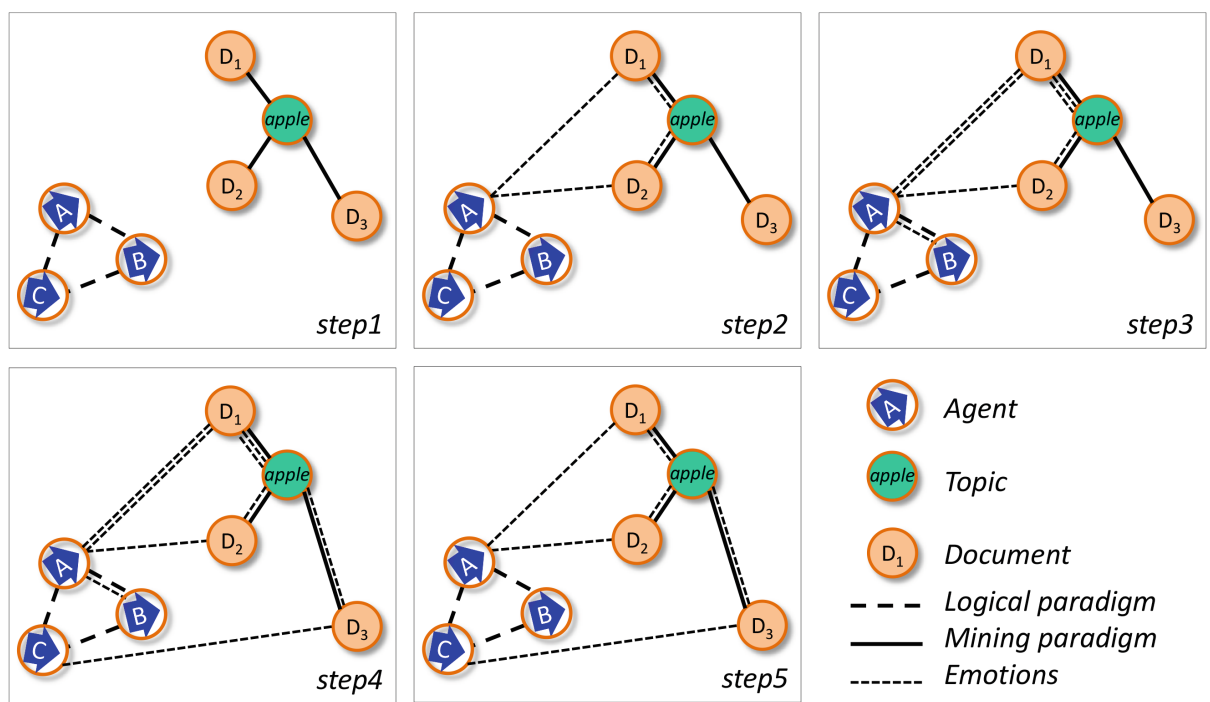

Fig. 2. The network of interlinked resources evolves along the attempts of the learners A, B and $\mathrm{C}$ to "catch" the topic 'apple' through accessing the modules $\mathrm{D}_{1}, \mathrm{D}_{2}$ or $\mathrm{D}_{3}$. (Color figure online)

$K G) . D_{1}, D_{2}$ and $D_{3}$ are documents that a mining algorithm has indexed by the topic/tag 'apple'. So, in Fig. 2 you notice the three types of resources: topics (one, in green: apple), documents (three, in orange: D1, D2, D3) and agents (three, in blue: A, B, C). In the example each of the three agents consider each other agent as trusted and adopts the same preferences so that we may say that there is a unique KM seen by each agent. This not necessarily true but simplifies once more the example without sacrificing the evidence of situations of potential serendipitous behavior.

Step2: $A$ is a calm person who has time; she browses through $D_{1}, D_{2}$ and $D_{3}$ and has a positive emotion about $D_{1}$ and $D_{2}$ (she likes both and finds them relevant with respect to 'apple'); the capture of this emotion results in linking $\mathrm{D}_{1}$ and $\mathrm{D}_{2}$ to her and reinforcing the links between the documents and the topic 'apple'. B is always in a hurry; he asks the KM the question "which is the shortest path between me and the topic "apple'?"? According to the paths in the diagram, he gets a double answer: B-A- $\mathrm{D}_{1}$-'apple' and $\mathrm{B}-\mathrm{A}-\mathrm{D}_{2}$-'apple'. B is exploiting the behavior of $\mathrm{A}$ in order to answer a question. This is already a source of potential "surprise". In general, the introduction of Agents in the KG and KM as first-class citizens is the major potential source of surprise and serendipity. There is a second major difference with traditional search engines: B (like A) can as well directly access D1, D2 and D3 by launching a query; but "trusts" the search done by $A$ reducing in this way the overload of the group of trusted Agents ( $A, B$ and $C$ ).

7 This is obviously an oversimplification: in the real prototype available, the KM is visualized and the notion of proximity (short path) is reified by real distances in the visualized graph. Another way to convey the same message without visualization is to give a list of answers in order of increasing distance. In this case the difference with Google's answers is evident. 
Step3: $\mathrm{B}$ has a positive emotion about $\mathrm{D}_{1}$ but not about $\mathrm{D}_{2}$; this results in reinforcing the path B-A-D - -'apple'. If he would ask his question now, he would then get only $\mathrm{D}_{1}$. The system's behavior depends on the history of interactions, adapts to the user's historical behavior.

Step4: C likes to explore; rather than taking a short path she browses through $\mathrm{D}_{1}, \mathrm{D}_{2}$ and $\mathrm{D}_{3}$ and has a positive emotion about $\mathrm{D}_{3}$ (she likes it and finds it relevant with respect to 'apple'); this results in linking $\mathrm{D}_{3}$ to her and reinforcing the linking between $\mathrm{D}_{3}$ and the topic 'apple'. At this stage, if A, B and C would ask for the shortest path to 'apple', they would respectively get $\mathrm{D}_{1}, \mathrm{D}_{1}$ and $\mathrm{D}_{3}$. Notice that these modifications occur as a result of asynchronous, unforeseeable Agent's interactions with the KM generated by the KG fed by three autonomous Human Agents that activate an Artificial Agent: the search engine. The example concerns a minimal number of resources; when the size of the resources increases significantly, we may envision the emergence of a "collective wisdom of the crowd" where the "crowd" is controlled by each one's preferences and perspectives, not by external algorithms.

Step5: B has read again D1 and is not fully satisfied by $\mathrm{D}_{1}$; he asks again for a short path: but in order to discover new sources of knowledge, he changes his perspective: he discards the viewpoints expressing his own emotions. The KM is dynamic, changes not only as a function of the peer's behavior but also our own history. This new

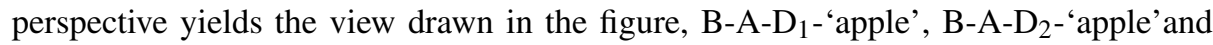
B-C- $\mathrm{D}_{3}$-'apple' have the same length i.e., $\mathrm{D}_{1}, \mathrm{D}_{2}$ and $\mathrm{D}_{3}$ are equidistant from him. He may now discard $\mathrm{D}_{1}$ (already visited) and $\mathrm{D}_{2}$ (already rejected) and study $\mathrm{D}_{3}$. B has $\boldsymbol{a}$ chance of discovering what Chas found interesting: serendipity may be triggered NOT as a consequence of an available "serendipitous object" proposed by an algorithm that finds something "similar in its essence" and "compatible (relevance) with the user" -as most recommender systems propose- but capitalizing on the autonomous behavior of trusted peers that may depend on much more sophisticated (and unforeseen) choices, previous knowledge, emotional states etc. For instance, if Agent $C$ is an artist, she may find D3 interesting and relevant for "apple” because D3 is a famous painting of Cezanne or Renoir, not a biologist's classification of diverse apple types (D1 for instance) or an cook's story of how to exploit apples for making cakes (D2 for instance).

Along the five steps of this imaginary case, the evolution of "knowledge paths" follows the metaphor of the selective reinforcement of neural beams [4], except that this reinforcement is not regulated by a single system of values, rather by a collaboration/competition between the three systems of values of A, B and C. The three co-learners learn as a whole, in a trans-disciplinary way: the dynamics are governed by emotions and topology, not by logics.

Emotions influence the KM that each "learner" sees, i.e.: the distance (s)he perceives from the available resources (Agents, Topics and Documents). This, however, occurs in the community of trusted agents, not in the single one. Each learner is influenced by the choices made by other agents, with an indirect effect on his/her exposure to unexpected resources (Agents, Documents, Topics) that may trigger his/her "new connection" (see the lamp in Fig. 1, often associated to Archimede's EUREKA!). 


\section{Conclusion}

We have presented our new paradigm/model/method/system/environment called ViewpointS and the opportunities offered with respect to possibly understand, forecast and foster serendipitous learning, a conjecture that we consider very likely to be true. This potential was not among the initial goals of the work, so we may state that it has been a serendipitous encounter. In this paper, we have made an effort to motivate retrospectively, on the basis of part of the available literature, the adequacy of our conjecture.

The foundations of our conviction reside on two properties that ViewpointS may include: the assessment of brain states relating to emotions, to be associated to single viewpoints, and the collective wisdom embodied in the model. These components have been described in fine detail in a toy example that has been annotated in order to unveil its potential application.

We conclude that such a proposal would not be acceptable just a few years ago when measurements of brain functions were much harder to assess and the Web did not have the size it has today ("big" data but also "big" people!).

Concerning the last phenomenon, we believe that the construction of a "protected knowledge space" such as the one envisaged by ViewpointS is today non just an issue of optimization and personalization of the access to information, but a real challenge for the defense of digital sovereignty from the control of external undesired or irrelevant agents.

Regarding the first - brain state measurements - we have been encouraged and fully convinced by the results of Conferences such as: [16] that show the current availability of equipment and of human expertise for assessing and exploiting brain state measurements in real time interactive applications including biofeedback.

\section{References}

1. Cerri, Stefano A., Lemoisson, P.: Tracing and enhancing serendipitous learning with viewpointS. BFAL 2017. LNCS (LNAI), vol. 10512, pp. 36-47. Springer, Cham (2017). https:// doi.org/10.1007/978-3-319-67615-9_3

2. Lemoisson, P., Surroca, G., Jonquet, C., Cerri, S.A.: ViewpointS: when social ranking meets the semantic web. In: FLAIRS 17 Conference Proceedings, Florida Artificial Intelligence Research Society Conference, North America, May 2017. https:/www.aaai.org/ocs/index. php/FLAIRS/FLAIRS17/paper/view/15432. Accessed: 17 Aug 2020

3. Lemoisson, P., Surroca, G., Jonquet, C., Cerri, S.A.: ViewPointS: capturing formal data and informal contributions into an evolutionary knowledge graph. Int. J. Knowl. Learn. 12(2), 119-145 (2018)

4. Edelman, G. M.: Neural Darwinism: The Theory of Neuronal Group Selection. Basic Books (1989)

5. Van Andel, P.: Serendipiteit: de paradox van de ongezochte vondst: 'toevallige' ontdekkingen, uitvindingen en creaties uit wetenschap, techniek en kunst, $\mathrm{PhD}$ thesis, Un. Groningen (1989), also published (in Dutch) as a book with Bert Andreae

6. Van Andel, P.: Anatomy of the unsought finding. serendipity: origin, history, domains, traditions, appearances, patterns and programmability. Br. J. Philos. Sci. 45(2), 631-648 (1994). Oxford University Press. http://www.jstor.org/stable/687687. (verified 8 March 2020) 
7. Corneli, J., et al.: Modelling serendipity in a computational context (2014). https://arxiv.org/ abs/1411.0440. (Submitted on 3 Nov 2014 (v1), last revised 30 Aug 2019 (v7))

8. Cerri, S.A., Loia, V., Maffioletti, S., Fontanesi, P., Bettinelli, A.: Serendipitous acquisition of web knowledge by agents in the context of Human Learning. In: THAI-ETIS, Varese, Italy; June 21-22, 1999, pp. 1-25 (1999)

9. Toms, E.G.: Serendipitous information retrieval. In: Proceedings of the First \{DELOS $\}$ Network of Excellence Workshop on Information Seeking, Searching and Querying in Digital Libraries, \{DELOS \} 2000, Zurich, Switzerland, December 11-12, 2000, ERCIM Workshop Proceedings, 01/W001, ERCIM (2000)

10. Makri, S., Blandford, A.: Coming across information serendipitously - Part 1: a process model. J. Doc. 68(5), 684-705 (2012). http://dx.doi.org/10.1108/00220411211256030

11. Makri, S., Tammera, T.M.: Serendipity in future digital information environments. In: Accidental Information Discovery, pp. 81-114. Chandos Publishing (2016). http://www.sciencedi rect.com/science/article/pii/B9781843347507000054. (verified 11 March 2020)

12. Weigl, D.M., Emsley, I., Page, K.R.: Serendipity in context: prioritised contextual browsing in large-scale digital libraries. In: Proceedings of the Association for Information Science and Technology, vol. 55, 1, pp. 544-553 (2018). https://doi.org/10.1002/pra2.2018.145055 01059. (verified 16 March 2020)

13. Chirita, C.: Modelling serendipity in a service-oriented context. In: AISB Symposium (2017). http://ccg.doc.gold.ac.uk/serendipitysymposium/. (verified 11 March 2020)

14. Kotkov, D., Veijalainen, J., Wang, S.: Challenges of serendipity in recommender systems. In: Majchrzak, T.A., Traverso, P., Monfort, V., Krempels, K.-H. (eds.) WEBIST 2016: Proceedings of the 12th International Conference on Web Information Systems and Technologies, vol. 2, pp. 251-256. SCITEPRESS, Setúbal (2016)

15. de Lima, A.P., Marques Peres, S.: Limits to Surprise in Recommender Systems. eprint $=$ $\{1807.03905\}$, archivePrefix $=\{$ arXiv $\}$, primaryClass $=\{$ cs.IR $\}(2018)$

16. Frasson, C., Kostopoulos, G.: Brain function assessment in learning. In: First International Conference, BFAL 2017, Patras, Greece, September 24-25, 2017, Proceedings. Lecture Notes in Artificial Intelligence, vol. 10512, pp. XV, 215. Springer International Publishing (2017)

17. Lemoisson, P. and Cerri, S.A.: ViewpointS: a Collective Brain. This volume

18. Lemoisson, P., Rakotondrahaja, C.M.H., Andriamialison, A.S.P., Sankar, H.A., Cerri, S.A.: VWA: viewpoints web application to assess collective knowledge building. In: Nguyen, N.T., Chbeir, R., Exposito, E., Aniorté, P., Trawiński, B. (eds.) ICCCI 2019. LNCS (LNAI), vol. 11683, pp. 3-15. Springer, Cham (2019). https://doi.org/10.1007/978-3-030-28377-3_1 


\section{Author Queries}

Chapter 14

\begin{tabular}{|c|l|c|}
\hline Query Refs. & Details Required & Author's response \\
\hline AQ1 & $\begin{array}{l}\text { This is to inform you that corresponding author has been identified } \\
\text { as per the information available in the Copyright form. }\end{array}$ & \\
\hline AQ2 & $\begin{array}{l}\text { Reference [7] is given in the list but not cited in the text. Please } \\
\text { cite in text or delete from the list. }\end{array}$ & \\
\hline
\end{tabular}

